\title{
Nonlinear Identification of an Anaerobic Digestion Process
}

\author{
Linda Brus \\ Systems and Control, Department of Information Technology, Uppsala University \\ P.O. Box 337, SE-751 05 Uppsala, SWEDEN
}

\begin{abstract}
Anaerobic digestion in bioreactors is an important technology for environmental friendly treatment of organic waste. To optimize and control such processes accurate dynamic models of the process are needed. Unfortunately, modeling of anaerobic digestion often results in high order nonlinear models with many unknown parameters, a fact that complicates controller design. This paper attempts to circumvent this problem, by application of new recursive system identification techniques, thereby radically reducing the degree of the models and the number of parameters. Experiments show that a second order nonlinear model is sufficient for accurate modeling of the system.
\end{abstract}

\section{INTRODUCTION}

With increased population and migration to urban areas the need for sustainable ways to manage the large amounts of waste produced increases. Some of the waste, e.g. domestic compost material, waste from food production, cattle and pig manure, and sludge from wastewater treatment, is of organic origin and is decomposed by microorganisms in nature. However, the amounts or concentrations associated with urban areas or large scale food production poses problems.

In a bioreactor, organic material can be decomposed by microorganisms in an anaerobic environment, producing methane (biogas) that can be used for fuel, and extracting nutrients that can be used for fertilization. As a bonus, the volume of waste used for landfill is reduced, and the amount of leachate from the landfill decreases.

To get an efficient digestion process the environment in the bioreactor needs to remain propitious for the microorganisms. This control problem is complicated by the sensitivity and complexity of the system as well as by the slow dynamics, which make pilot scale experiments time consuming. A model of the process can to some extent replace pilot scale experiments. However, if the model is to be used for control purposes it is preferable if the model structure is as simple as possible without losing important aspects of the system dynamics. The anaerobic digestion process is nonlinear which must also be considered in modeling and controller design. Examples of previous studies of nonlinear modeling and control of anaerobic digestion and biogas production include [1], [2], and [3]. In [1] an adaptive controller based on a simple model of anaerobic digestion is proposed, in [2] a procedure for model structure selection is considered, and [3] treats control design based on a grey-box model of a biogas tower reactor.

In this paper modeling by means of system identification is applied. Such modeling methods are often classified as greybox (semi-physical), or black-box (non-physical). In grey- box modeling a priori knowledge of the system dynamics is included in the model structure, followed by estimation of a number of unknown model parameters using measured data. Examples of grey-box modeling include [4]. In blackbox identification no previous knowledge of the system is required, which can be an advantage if information of the system dynamics is limited. An other advantage can be avoiding to get models that depend on a large number of parameters, which may be the case if detailed grey-box modeling of a complex system is performed. However, the black-box modeling involves the problem of choosing a suitable model structure.

A recursive prediction error method (RPEM) for solving nonlinear identification problems with a restricted black-box parameterization of a state space ODE was described in [5], [6]. The model exploits a multi-variable polynomial in one right-hand side component of the ODE, which offers flexibility in terms of application areas. One advantage is that the polynomial form enables identification using relatively few parameters. The model structure also offers a possibility to, locally, model systems with a more complex right-hand side structure of the ODE.

In this paper the main purpose is to apply the above RPEM to simulated data from a biogas reactor model. The contribution of the paper include showing that nonlinear black-box identification can be used for modeling of biogas production with good results. A second order nonlinear model with eight parameters is sufficient to describe the system. A second order linear model, however, fails to describe some of the system dynamics. The two state, eight parameter model is substantially less complex than the 34 state grey-box model from which the data was obtained. This reduction in model complexity greatly simplifies e.g. the issue of control design for the biogas reactor. The more complex grey-box model has been evaluated in e.g. [7].

This paper is organized as follows. In section II the anaerobic digestion data is described. Section III presents the model parameterization, followed by experiments in section IV. Finally, the conclusions can be found in section V.

\section{ANAEROBIC DIGESTION}

Biogas production has been introduced to treat organic waste, domestic as well as industrial. In the anaerobic environment of a biogas reactor microorganisms decompose organic material and produce hydrocarbons that can be used as an energy source. 

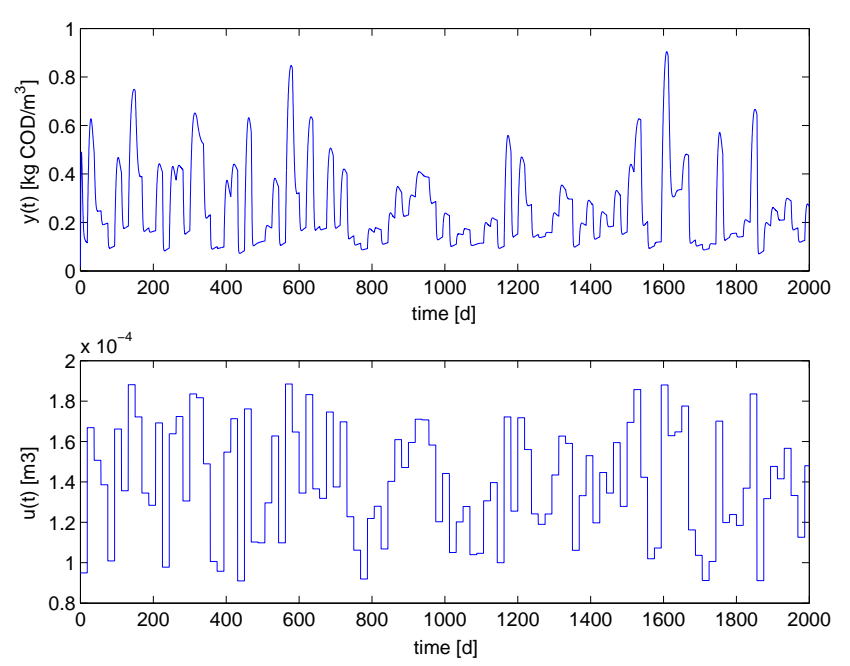

Fig. 1. Simulation data from the anaerobic digestion model (ADM1), propionate concentration (top), and substrate input (bottom).

The experimental data used in this paper (see Fig. 1) comes from simulations [8] using the IWA ADM1, an anaerobic digestion model presented by the International Water Association [9]. The model is of grey-box type, estimating the concentration of a substantial number of chemical compounds as well as the amount of biomass in the reactor. Implemented as a set of differential and algebraic equations, the model consists of 26 dynamic state concentration variables, and 8 algebraic variables per reactor vessel or element. If implemented as differential equations only, the model has 32 dynamic concentration state variables. This rather complex model can be used for detailed estimation of different aspects of the decomposition process.

Since the time constants of microbial growth in an anaerobic digestion reactor are large, the model offers an alternative to long and costly experiments in a pilot scale reactor. It also enables the input signal to be persistently exciting in terms of frequency and amplitude, which is crucial to the nonlinear identification, without having to consider the environmental problems of varying process performance, associated with physical experiments.

In the ADM1 simulation used in this paper the microorganisms are fed with domestic waste, but the initial values are adjusted to digestion of excess waste water sludge. It takes a while for the process to stabilize after the change of conditions, and therefore the first 100 samples (25 days) were not used for the experiments.

To obtain better conditions for identification the data was rescaled, the input signal with a factor $10^{4}$ and the output with a factor 10 . However, in all plots the input and output (ADM1 generated as well as modeled) are in the original scale.

\section{MODEL AND ALGORITHM}

\section{A. Model}

The identification was performed with a recursive prediction error algorithm with a restricted black-box parameteriza- tion. To describe the identification model, which is of output error $(\mathrm{OE})$ type, introduce the input vector

$$
\boldsymbol{u}(t)=\left(u_{1}(t) \ldots u_{1}^{\left(n_{1}\right)}(t) \ldots u_{k}(t) \ldots u_{k}^{\left(n_{k}\right)}(t)\right)^{T}
$$

and the state vector

$$
\boldsymbol{x}(t)=\left(\begin{array}{llll}
x_{1}(t) & x_{2}(t) \quad \ldots \quad x_{n}(t)
\end{array}\right)^{T}
$$

The superscript $j$ of $u_{i}^{(j)}$ denotes the $j^{t h}$ derivative of the $i^{\text {th }}$ input signal. The algorithm is based on the state space model

$$
\begin{aligned}
& \boldsymbol{x}^{(1)}=\left(\begin{array}{c}
x_{1}^{(1)} \\
\vdots \\
x_{n-1}^{(1)} \\
x_{n}^{(1)}
\end{array}\right)=\left(\begin{array}{c}
x_{2} \\
\vdots \\
x_{n} \\
f(\boldsymbol{x}, \boldsymbol{u}, \boldsymbol{\theta})
\end{array}\right) \\
& y(t)=\left(\begin{array}{llll}
1 & 0 & \ldots & 0
\end{array}\right) \boldsymbol{x}
\end{aligned}
$$

where $\boldsymbol{\theta}$ is the unknown parameter vector containing $n_{\theta}$ components. The right-hand side of (4) can be exchanged for any set of known nonlinear vector functions to expand the model to a general multiple output model (cf. [5]).

By limiting the nonlinearity to entering equation (3) in one of the right-hand side components only, overparameterization can be avoided and the model structure complexity kept relatively low. Yet, the state space form (3)-(4) provides a possibility to model a wide variety of right-hand side components through the function $f$. In fact, it is proven in [5] and the references therein that the model can (locally) describe systems with arbitrary right-hand side structures.

$f(\boldsymbol{x}, \boldsymbol{u}, \boldsymbol{\theta})$ is chosen to be of polynomial form

$$
\begin{aligned}
& f(\boldsymbol{x}, \boldsymbol{u}, \boldsymbol{\theta})= \\
& =\sum_{i_{x_{1}}=0}^{I_{x_{1}}} \ldots \sum_{i_{x_{n}}=0}^{I_{x_{n}}} \sum_{i_{u_{1}}=0}^{I_{u_{1}}} \ldots \sum_{i_{u_{1}\left(n_{1}\right)}=0}^{I_{\left.u_{1}^{(n}\right)}} \ldots \sum_{i_{u_{k}}=0}^{I_{u_{k}}} \ldots \sum_{i_{u_{k}^{\left(n_{k}\right)}}=0}^{I_{u_{k}}^{I_{k}\left(n_{k}\right)}}
\end{aligned}
$$

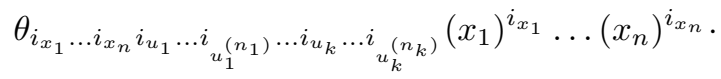

$$
\begin{aligned}
& \cdot\left(u_{1}\right)^{i_{u_{1}}} \ldots\left(u_{1}^{\left(n_{1}\right)}\right)^{i u_{1}^{\left(n_{1}\right)}} \ldots\left(u_{k}\right)^{i_{u_{k}}} \ldots\left(u_{k}^{\left(n_{k}\right)}\right)^{i u_{k}^{\left(n_{k}\right)}}= \\
& =\boldsymbol{\varphi}^{T}(\boldsymbol{x}, \boldsymbol{u}) \boldsymbol{\theta}
\end{aligned}
$$

For example, a simple second order model of this representation would be with two states, $x_{1}$ and $x_{2}$ (corresponding to the output signal, and its derivative, cf. equation (4)) and one input signal, $u$ where $n_{1}=0$, (the differential equation does not depend on derivatives of the input signal in this case). This means that $n_{\theta}=8$,

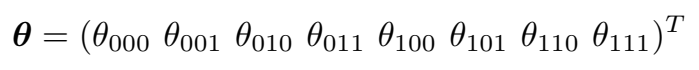

and

$$
\boldsymbol{\varphi}=\left(\begin{array}{llllllll}
1 & u & x_{2} & x_{2} u & x_{1} & x_{1} u & x_{1} x_{2} & x_{1} x_{2} u
\end{array}\right)^{T}
$$

Remark 1: A discussion on the consequenses of the use of input signal derivatives appears in [5]. Note that no input signal derivatives are used in this paper. 


\section{B. Discretization}

To be able to formulate the model as an RPEM algorithm it needs to be discretized. Applying the Euler integration method to (3), and using the relations (4) and (5) the following discrete time model is obtained

$$
\begin{aligned}
& \left(\begin{array}{c}
x_{1}\left(t+T_{S}, \boldsymbol{\theta}\right) \\
\vdots \\
x_{n-1}\left(t+T_{S}, \boldsymbol{\theta}\right) \\
x_{n}\left(t+T_{S}, \boldsymbol{\theta}\right)
\end{array}\right)= \\
& \left(\begin{array}{c}
x_{1}(t, \boldsymbol{\theta}) \\
\vdots \\
x_{n-1}(t, \boldsymbol{\theta}) \\
x_{n}(t, \boldsymbol{\theta})
\end{array}\right)+T_{S}\left(\begin{array}{c}
x_{2}(t, \boldsymbol{\theta}) \\
\vdots \\
x_{n}(\boldsymbol{\theta}) \\
\boldsymbol{\varphi}^{T}(\boldsymbol{x}, \boldsymbol{u}, \boldsymbol{\theta}) \boldsymbol{\theta}
\end{array}\right) \\
& y(t, \boldsymbol{\theta})=\left(\begin{array}{llll}
1 & 0 & \ldots & 0
\end{array}\right) \boldsymbol{x}(t, \boldsymbol{\theta})
\end{aligned}
$$

\section{RPEM}

From the discretized model an RPEM can now be formulated. The identification is performed using an output error approach, and the covariance matrix of the measurement disturbances is estimated on-line. The construction of the algorithm follows the standard approach of [10]. The RPEM is given by

$$
\begin{aligned}
& \varepsilon(t)=y_{m}(t)-y(t) \\
& \boldsymbol{\Lambda}(t)=\boldsymbol{\Lambda}\left(t-T_{S}\right)+\frac{\mu(t)}{t}\left(\varepsilon(t) \varepsilon^{T}(t)-\boldsymbol{\Lambda}\left(t-T_{S}\right)\right) \\
& \boldsymbol{R}(t)=\boldsymbol{R}\left(t-T_{S}\right)+\frac{\mu(t)}{t}\left(\boldsymbol{\psi}(t) \boldsymbol{\Lambda}^{-1}(t) \boldsymbol{\psi}^{T}(t)-\boldsymbol{R}\left(t-T_{S}\right)\right) \\
& \hat{\boldsymbol{\theta}}(t)=\left[\hat{\boldsymbol{\theta}}\left(t-T_{S}\right)+\frac{\mu(t)}{t} \boldsymbol{R}^{-1}(t) \boldsymbol{\psi}(t) \boldsymbol{\Lambda}^{-1}(t) \boldsymbol{\varepsilon}(t)\right]_{D_{M}} \\
& \left(\begin{array}{c}
x_{1}\left(t+T_{S}\right) \\
\vdots \\
x_{n-1}\left(t+T_{S}\right) \\
x_{n}\left(t+T_{S}\right)
\end{array}\right)= \\
& =\left(\begin{array}{c}
x_{1}(t) \\
\vdots \\
x_{n-1}(t) \\
x_{n}(t)
\end{array}\right)+\alpha T_{S}\left(\begin{array}{c}
x_{2}(t) \\
\vdots \\
x_{n}(t) \\
\varphi^{T}(t) \hat{\boldsymbol{\theta}}(t)
\end{array}\right) \\
& \boldsymbol{y}\left(t+T_{S}\right)=\left(\begin{array}{llll}
1 & 0 & \ldots & 0
\end{array}\right) \boldsymbol{x}\left(t+T_{S}\right) \\
& \frac{d \boldsymbol{\varphi}}{d x_{i}}(t)=\left(\mathbf { 0 } ^ { T } 1 u _ { k } ^ { ( n _ { k } ) } ( t ) \ldots \left(\left(x_{i+1}(t)\right)^{I_{x_{i+1}}} \ldots\right.\right. \\
& \ldots\left(x_{n}(t)\right)^{I_{x_{n}}}\left(u_{1}(t)\right)^{I_{u_{1}}} \ldots\left(u_{k}^{\left(n_{k}\right)}(t)\right)^{I_{u_{k}}^{\left(n_{k}\right)}} \\
& \left.\left.2 x_{i}(t) \quad 2 x_{i}(t) u_{k}^{\left(n_{k}\right)}(t) \ldots\right)\right), \quad i=1, \ldots, n \\
& \frac{d \boldsymbol{\varphi}}{d \boldsymbol{x}}(t)=\left(\begin{array}{c}
\frac{d \boldsymbol{\varphi}}{d x_{1}}(t) \\
\vdots \\
\frac{d \boldsymbol{\varphi}}{d x_{n}}(t)
\end{array}\right)
\end{aligned}
$$

$$
\begin{aligned}
& \left(\begin{array}{c}
\frac{d x_{1}}{d \boldsymbol{\theta}}\left(t+T_{S}\right) \\
\vdots \\
\frac{d x_{n-1}}{d \boldsymbol{\theta}}\left(t+T_{S}\right) \\
\frac{d x_{n}}{d \boldsymbol{\theta}}\left(t+T_{S}\right)
\end{array}\right)=\left(\begin{array}{c}
\frac{d x_{1}}{d \boldsymbol{\theta}}(t) \\
\vdots \\
\frac{d x_{n-1}}{d \boldsymbol{\theta}}(t) \\
\frac{d x_{n}}{d \boldsymbol{\theta}}(t)
\end{array}\right)+\alpha T_{S} \\
& \left(\begin{array}{c}
\frac{d x_{2}}{d \boldsymbol{\theta}}(t) \\
\vdots \\
\frac{d x_{n}}{d \boldsymbol{\theta}}(t) \\
\left.\boldsymbol{\varphi}^{T}(t)+\hat{\boldsymbol{\theta}}(t)\left(\frac{d \boldsymbol{\varphi}}{d \boldsymbol{x}}(t)\right){ }^{\left(\frac{d x_{1}}{d \boldsymbol{\theta}}(t)\right)^{T}} \ldots\left(\frac{d x_{n}}{d \boldsymbol{\theta}}(t)\right)^{T}\right)
\end{array}\right) \\
& \boldsymbol{\psi}\left(t+T_{S}\right)=\left(\begin{array}{llll}
1 & 0 & \ldots & 0
\end{array}\right) \frac{d \boldsymbol{x}}{d \boldsymbol{\theta}}\left(t+T_{S}\right)
\end{aligned}
$$

Here $\varepsilon(t)$ is the prediction error, $\boldsymbol{\Lambda}(t)$ is the running estimate of the covariance matrix of the measurement disturbance, $\mu(t) / t$ is the gain sequence, $\boldsymbol{R}(t)$ is the running estimate of the Hessian, and $\psi(t)$ is the gradient of the output prediction with respect to the parameter vector. The gradient is determined by dynamic recursion, using the dynamics from the linearized state space model of the system. $D_{M}$ is the set of parameter estimates that give stable models, and is introduced to ensure model stability. $D_{M}$ is determined by linearization. $\alpha$ is a scaling factor applied to the sampling period, see [6] for further details.

\section{Model characteristics and algorithmic properties}

The main advantage of the model used in this paper is that it enables a black-box approach for a nonlinear identification problem. The restricted black-box parameterization of the state space ODE, with a polynomial model of one right-hand side of the ODE, avoids inherent overparameterization. In addition the polynomial can, locally, model more complicated nonlinearities in the right-hand side of the ODE [6]. Due to the generality of a black-box approach, the model could be used for various applications within many engineering fields.

Certain conditions may, however, cause problems with the identification. For example, if the relative sizes of the state vector components differ a lot, the polynomial elements will differ in magnitude too. This may cause numerical problems in the RPEM algorithm. The scaling factor $\alpha$ in (10) can be applied to the sampling period to improve the conditioning of the identification problem, see [6] for further details.

\section{EXPERIMENTS}

\section{A. Order selection and data properties}

Identification experiments with the biogas reactor data have been performed using the model and algorithm described by equations (3)-(7), and (10), respectively. Step responses from the ADM1 indicate that the system needs to be modeled as $2^{\text {nd }}$ order, or more. Hence, the approach has been to make identification experiments on a basic second order model structure, and then use it as reference when changing the polynomial degree of the input.

As in linear identification it is important to use persistently exciting inputs when collecting data, in order not to miss important information on the system. Hence, the input needs to have a varying frequency content. Furthermore, since a nonlinear relation between inputs and output is assumed, 
a variation of input amplitudes is required to identify the nonlinear dynamics. In the experiments described in this paper the input signal is chosen as a PRBS with varying amplitudes, see Fig. 1. The steps of various amplitudes provides information on the static behavior of the system. The data consists of an input signal, which corresponds to the substrate volume added to the process $\left(\mathrm{m}^{3}\right)$, and an output that corresponds to the propionate concentration in the reactor $\left(\mathrm{kgCOD} / \mathrm{m}^{3}\right)$. The sampling period is 6 hours (0.25 days).

\section{B. Initialization and algorithm tuning - general considera- tions}

When initializing the RPEM algorithm, the choice of initial parameters, $\theta_{0}$, and the magnitude of the Hessian, $R_{0}$, are of great importance for the result of the identification. If $\theta_{0}$ is chosen unfavorably the algorithm may not converge to a stable parameter set. The size of the Hessian, $R$, determines how fast the parameters change, and with a badly chosen $R_{0}$, the algorithm may take very long to converge, or cause unstable parameter estimates.

In the experiments the initial parameter vector, $\theta_{0}$, was chosen to correspond to a stable linear system. Attempts to use a linear $\theta_{0}$ with a pole placement that would give a similar step response as the nonlinear ADM1 generated data, resulted in instability after a few iterations with the RPEM. A $\theta_{0}$ with more stable poles gave better results.

In models with a large number of $\theta$ parameters, it takes longer before all parameters have responded to system dynamics and converged. It can then be necessary to adjust the parameter updating gain, $\frac{\mu(t)}{t} R^{-1}(t) \psi(t) \Lambda^{-1}(t)$. Since the parameter updating gain is proportional to $\mu(t)$ and $R^{-1}$ the convergence speed can be increased by increasing $\mu(t)$ or reducing $R$. However, if the parameter updating gain is chosen too large there is an increased risk of numerical problems. For the experiments in this paper the default value of the initial Hessian $R(0)$ has been chosen as the identity matrix of dimension $n_{\theta} . \mu(t)$ was chosen as a standard updating function $\bar{\mu}(t)$ that tends to 1 exponentially. In addition there is a limitation factor $\frac{t}{t+\mu_{0}}$, where $\mu_{0}$ is chosen large, that makes $\mu(t)$ small for small $t$. The gain is defined as $\frac{\mu(t)}{t}=\frac{1}{t+\mu_{0}} \bar{\mu}(t)$.

An other important tuning parameter is the scaling factor of the sampling period. If the states differ significantly in magnitude the identification problem will be badly conditioned, and numerical problems will occur. By scaling the sampling period of the model, the eigenvalues of the Hessian can be adjusted (cf. [6]), and convergence speed will be improved. This way parameter sets that would otherwise lead to divergence and instability may converge. Similarly, the gain can be tuned to obtain more favorable conditions for convergence.

Though not treated in this paper, it is worth mentioning that other ways of increasing model complexity to catch unmodeled system dynamics could be to increase the model order, or the polynomial degree of the states. Both methods may, however, introduce convergence problems and increase
TABLE I

INITIALIZATION VALUES FOR THE DIFFERENT NONLINEAR TEST CASES. $i_{u}$ IS THE DEGREE OF THE INPUT IN THE POLYNOMIAL, $\theta_{u}$ IS THE INITIAL VALUE OF THE PARAMETER CORRESPONDING TO THE LINEAR INPUT TERM (SIMILARLY FOR $\theta_{x_{2}}$ AND $\theta_{x_{1}}$ ). $\alpha$ IS THE SCALING FACTOR OF THE SAMPLING TIME, $R_{0}=r I_{n_{\theta}}$, WHERE $I_{n_{\theta}}$ IS THE $n_{\theta} \times n_{\theta}$ IDENTITY MATRIX, IS THE INITIAL HESSIAN, AND $n_{\theta}$ IS THE NUMBER OF ELEMENTS IN THE PARAMETER VECTOR $\theta$

\begin{tabular}{|c||c|c|c||c||c||c|}
\hline$i_{u}$ & $\theta_{u}$ & $\theta_{x_{2}}$ & $\theta_{x_{1}}$ & $\alpha$ & $r$ & $n_{\theta}$ \\
\hline 1 & 0.1 & -1 & -1.5 & 1 & 10 & 8 \\
2 & 0.1 & -1.5 & -0.5 & 1 & 10 & 12 \\
3 & 0.1 & -1 & -1.5 & 0.4 & 2 & 16 \\
4 & 0.1 & -1 & -1.5 & 0.4 & 5 & 20 \\
\hline
\end{tabular}

the risk of instability. In general increasing the degree of the states poses more of a problem than increasing the degree of the input, since the model involves the derivatives of the states.

\section{Identification results}

Before running the recursive algorithm on the dataset an initial parameter vector is required. Chosing suitable initial values for the $\theta$ vector is not trivial. Preferably the vector should be chosen close to the optimal values, to ensure convergence, and avoid local minima. However, the optimal values are, obviously, unknown. If initialized wrongly, the model will produce unstable parameter estimates or converge to a local minimum.

The experiments presented in this paper were based on a simple second order model, which was repeatedly extended by incresing the polynomial order of the input, $i_{u}$ (cf. (5)). For comparison the identification was also performed on a model containing only the linear parameters of the second order system, i.e. $\varphi=\left(\begin{array}{llll}1 & u & x_{2} & x_{1}\end{array}\right)$ (see Fig.2).

Initialization of the different test cases were all done starting with all nonlinear parameter values set to zero. The linear parameters were then adjusted to obtain a stable parameter estimation that converges. For example, the basic nonlinear model with a first degree input $\left(i_{u}=1\right)$ was

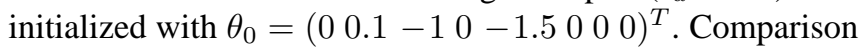
to (6)-(7) shows that the nonzero elements correspond to the $u, x_{2}$, and $x_{1}$ parameters respectively. To obtain convergence the initial values of the Hessian needed adjustment. Table I shows the initial values for the linear parameter elements, the sampling scale factor, and the initial Hessian.

Figure 2 shows a close up of the simulation results from the linear model experiment. It is clear that the linear model structure only manages to describe a fraction of the system dynamics. The basic nonlinear second order model (see Fig. 3) performes radically better, and explains most of the output variations The parameter estimates converge and behave nicely, which can be seen in Fig. 4.

Figures 5, 6, and 7, show the results of simulations with $i_{u}=2, i_{u}=3$, and $i_{u}=4$, respectively. To highlight the difference in model performance the plots only cover the time between day 500 and 1500 . All three alternatives 
manage to model the main dynamics, but fail somewhat to describe some of the overshoot that occurs at large changes in input substrate. It is also worth mentioning that the higher the degree of $\mathrm{u}$, the more parameters, and thereby an increased difficulty to get the model to converge. With increased model complexity the algorithm becomes more sensitive to the choise of initial parameters as well as to scaling and gain. The $3^{\text {rd }}$ and $4^{\text {th }}$ degree models also show a tendency to oscillate, if the RPEM parameters are not chosen carefully.

For the biogas data the simulations shows high accuracy already at models with a low polynomial degree of the input signal. The improvement compared to the linear model is substantial. Model accuracy improves slightly as $i_{u}$ increases, however, the low degree model (with $i_{u}=$ 1) is sufficient to describe most of the system dynamics. The model structure opens for identification using live data, which would be very complicated with a grey-box model with a large number of parameters. The more complex greybox model is likely to be harder to tune and stability issues may easily occur due to the large number of parameters. A low degree model also simplifies control design significantly, as the number of parameters is small.

Interesting topics for future research include applying the obtained model to live data from a full scale biogas reactor. Further, designing nonlinear controlers based on the model would be of great interest. The model structure, based on the polynomial form, enables application to a number of control strategies, see e.g. [11]. Finally the obtained controllers could be evaluated when applied to a real biogas reactor. Improvements to the RPEM include developing an initialization method, to simplify the choice of initial parameters for complex model structures. It would also be of interest to study the behaviour of higher order models, i.e. more states and derivatives of the input signals included in the nonlinear model.

The software implementation of the algorithm that was used for the identification experiments is described in [12], and can be downloaded from http://www.it.uu.se/research/reports.

\section{CONClusions}

The identification experiments in this paper show that an anaerobic digestion process can be modeled, obtaining good results, with as little as eight parameters. This relatively uncomplicated model could greatly simplify the issue of controller design for the complex biological system in a biogas reactor.

The identification method was an RPEM based on a restricted black-box state space model. Different second order model structures were used for the identification. A linear model was insufficient to describe the system dynamics, whereas nonlinear models that describe most of the dynamics could be found for models with input polynomial degree of 1 to 4 . The first degree input model was very accurate, and contains few parameters compared to $e$.g. the corresponding grey-box model. The simple model structure and the low

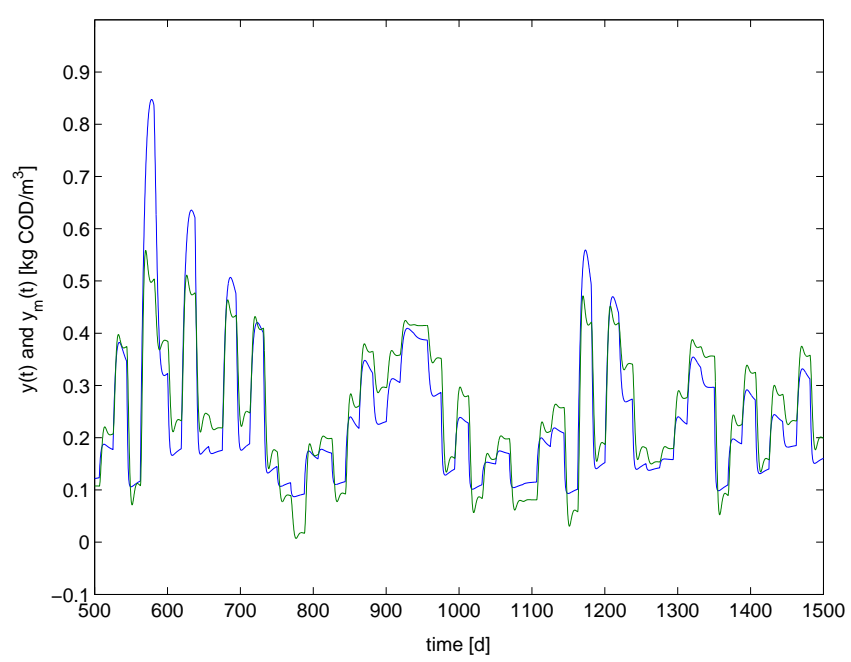

Fig. 2. ADM1 (blue) and simulated (green) propionate concentration for the linear $2^{\text {nd }}$ order model, from day 500 to day 1500 .

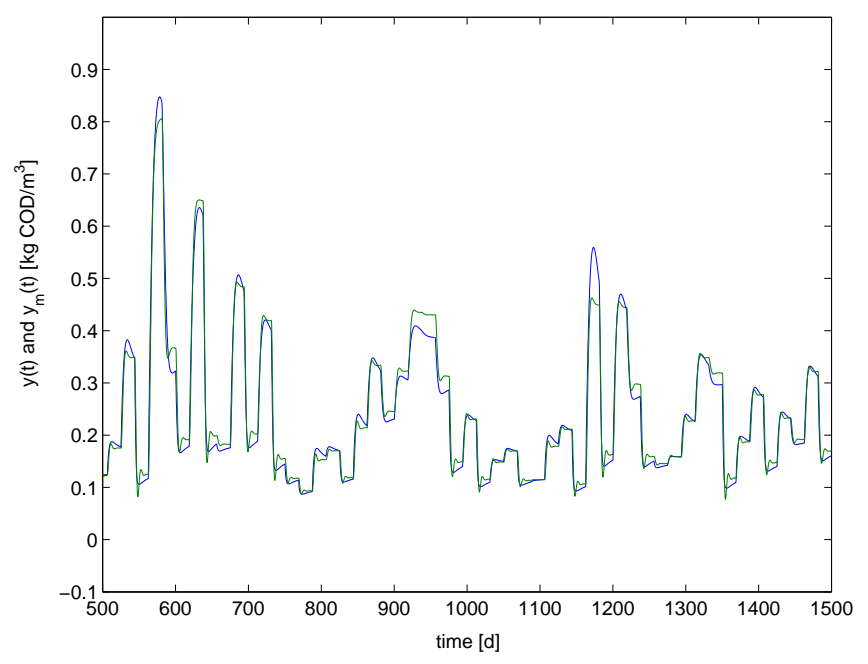

Fig. 3. ADM1 (blue) and simulated (green) propionate concentration for the $2^{\text {nd }}$ order model with $i_{x_{1}}=1, i_{x_{2}}=1$, and $i_{u}=1$.

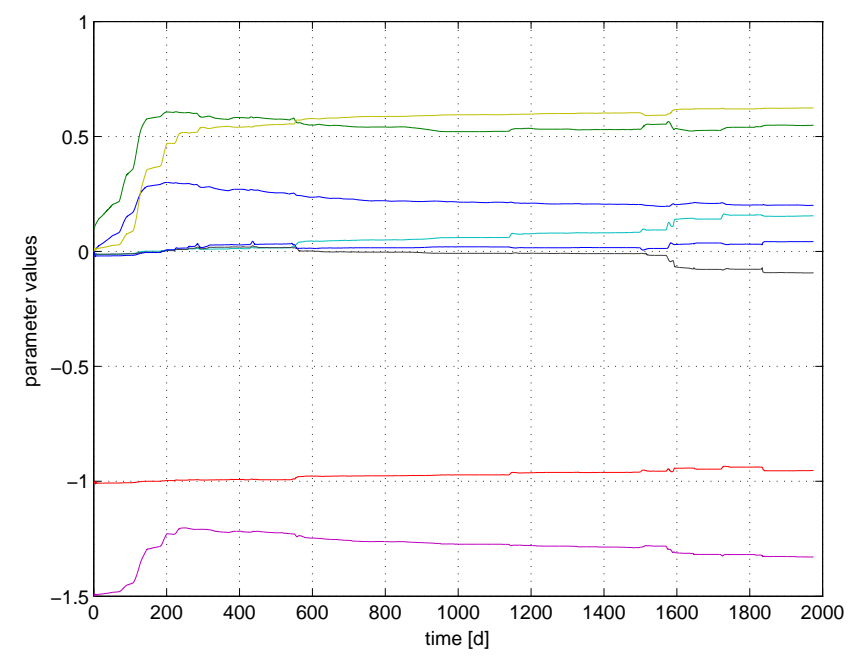

Fig. 4. Parameter estimates for the basic nonlinear $2^{\text {nd }}$ order model, $i_{x_{1}}=1, i_{x_{2}}=1$, and $i_{u}=1$ 


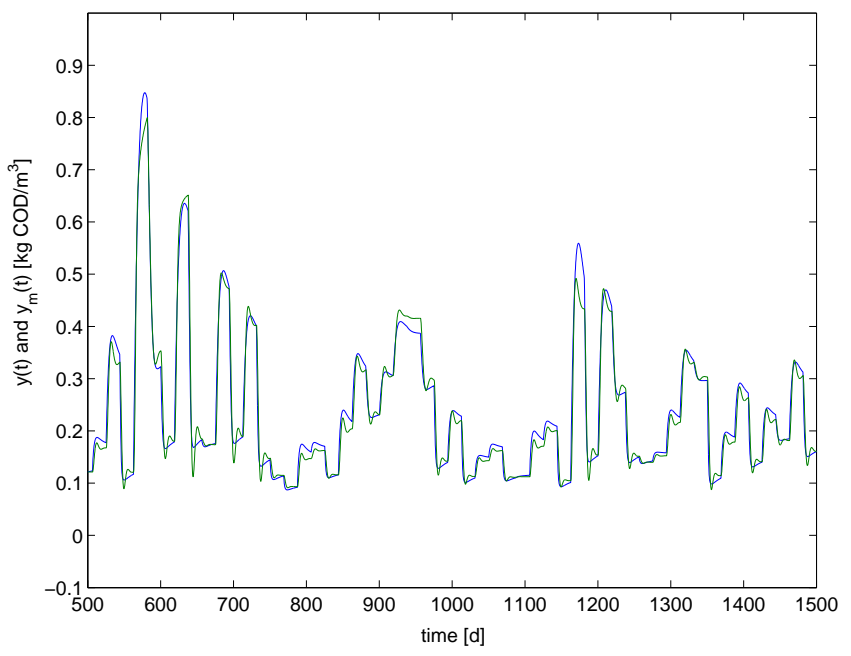

Fig. 5. ADM1 (blue) and simulated (green) propionate concentration for the $2^{\text {nd }}$ order model with $i_{x_{1}}=1, i_{x_{2}}=1$, and $i_{u}=2$.

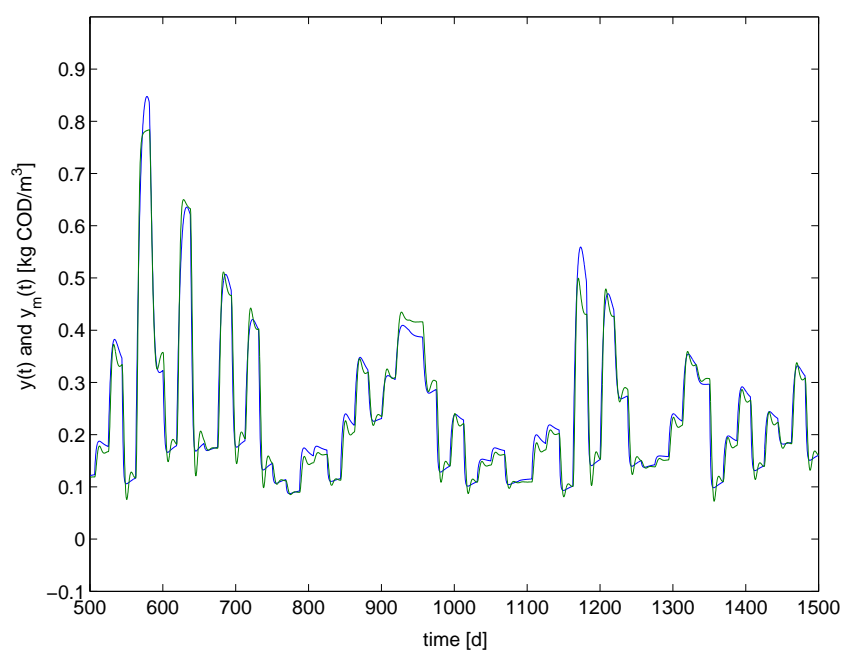

Fig. 6. ADM1 (blue) and simulated (green) propionate concentration for the $2^{\text {nd }}$ order model with $i_{x_{1}}=1, i_{x_{2}}=1$, and $i_{u}=3$.

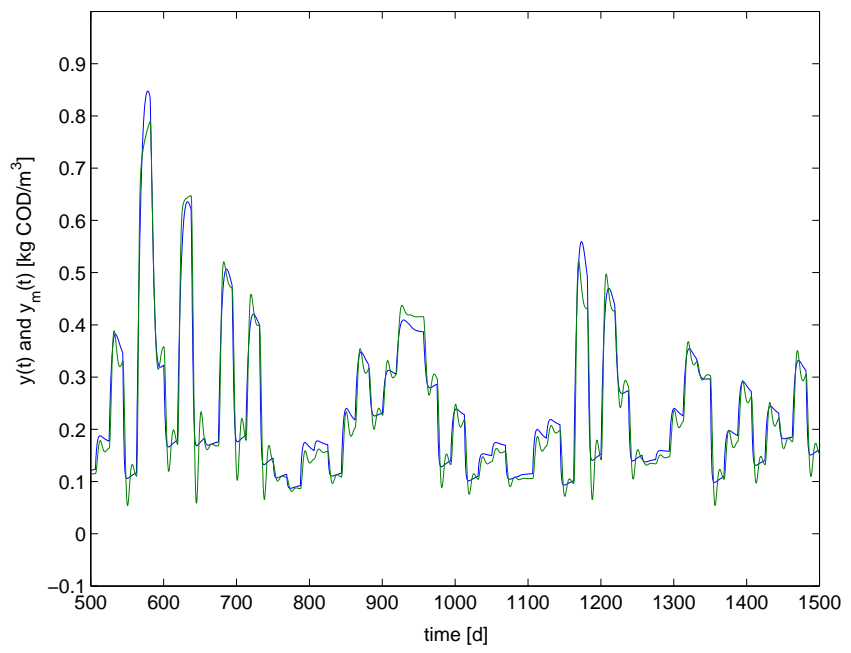

Fig. 7. ADM1 (blue) and simulated (green) propionate concentration (top), and substrate volume (bottom) for the $2^{\text {nd }}$ order model with $i_{x_{1}}=1$, $i_{x_{2}}=1$, and $i_{u}=4$. number of parameters open for direct application to live data and is believed to significantly simplify controller design.

Interesting topics for further research would be to study the model behaviour when applied to measured data from a biogas reactor, to design nonlinear controllers based on the model, and eventually to evaluate the controller performance on a real reactor. As for the algorithm future work could include developing an initialization method for the RPEM, and to study the behaviour of higher order models, i.e. more states and derivatives of the input signals included in the nonlinear model.

\section{ACKNOWLEDGEMENTS}

The author wishes to thank Prof. Torbjörn Wigren for supervision and valuable comments, and Mr. Mikael Hansson for providing the data from the Anaerobic Digestion Model No.1. This work has been financially supported by the EC 6th Framework programme as a Specific Targeted Research or Innovation Project (HipCon, Contract number NMP2-CT2003-505467). Note that the European Community is not liable for any use that may be made of the information contained herein.

\section{REFERENCES}

[1] J. Alvarez-Ramirez, R. Femat, O. Monroy, and F. Cuervo, "An adaptive control strategy for anaerobic digester," in Proc. of IEEE Int. Conference on Control Applications, 1996.

[2] J. E. Haag, A. V. Wouwer, and I. Queinnec, "Macroscopic modelling and identification of an anaerobic waste treatment process," Chemical Engineering Science, vol. 58, pp. 4307-4316, 2003.

[3] M. Pahl and J. Lunze, "Dynamic modelling of a biogas tower reactor," Chemical Engineering Science, vol. 53, pp. 995-1007, 1998.

[4] T. Bohlin, "A case study of grey box identification," Automatica, vol. 30, pp. 307-318, 1994.

[5] T. Wigren, "Recursive identification based on nonlinear state space models applied to drum-boiler dynamics with nonlinear output equations," in Proceedings of ACC 2005, Portland, Oregon, U.S.A, 2005.

[6] _ - "Scaling of the sampling period in nonlinear system identification," in Proceedings of ACC 2005, Portland, Oregon, U.S.A, 2005.

[7] M. Hansson, S. Hidén, . Nordberg, and B. Carlsson, "Application of the iwa anaerobic digestion model no1 (adm1) to a lab-scale digester operated on the source-sorted fraction of municipal solid waste," in Proceedings of the 10th IWA World Congress on Anaerobic Digestion, Montreal, Canada, 2004.

[8] M. Hansson, 2004, personal communication.

[9] IWA, "Anaerobic Digestion Model no.1 (ADM1)," Scientific and Technical Report No. 13, 2002, iWA Printing, London.

[10] L. Ljung and T. Söderström, Theory and practice of recursive identification. Cambridge, MA: M.I.T. Press, 1983.

[11] H. K. Khalil, Nonlinear systems, 2nd ed. Upper Saddle River, NJ: Prentice-Hall, Inc., 1996.

[12] T. Wigren, "A matlab software package for recursive identification and scaling using a structured nonlinear black box model," Systems and Control, Department of Information Technology, Uppsala University, Uppsala, Tech. Rep. 2005-002, 2005. 